\title{
Nanofabrication using home-made RF plasma coupled chemical vapour deposition system
}

\author{
Si Ci Ong \\ River Valley High School, 6 Boon Lay Avenue, Singapore 649961 \\ ongsici@gmail.com \\ Usman Ilyas \\ National Institute of Education, Nanyang Technological University, \\ 1 Nanyang Walk, Singapore 637616, Department of Physics, University of Engineering \& Technology \\ Lahore 54890, Pakistan \\ iusman82@gmail.com \\ Rajdeep Singh Rawat \\ National Institute of Education, Nanyang Technological University, \\ 1 Nanyang Walk, Singapore 637616 \\ rajdeep.rawat@nie.edu.sg \\ Published 13 August 2014
}

\begin{abstract}
Zinc oxide, $\mathrm{ZnO}$, a popular semiconductor material with a wide band gap (3.37 eV) and high binding energy of the exciton $(60 \mathrm{meV})$, has numerous applications such as in optoelectronics, chemical/biological sensors, and drug delivery. This project aims to (i) optimize the operating conditions for growth of $\mathrm{ZnO}$ nanostructures using the chemical vapor deposition (CVD) method, and (ii) investigate the effects of coupling radiofrequency (RF) plasma to the CVD method on the quality of $\mathrm{ZnO}$ nanostructures. First, $\mathrm{ZnO}$ nanowires were synthesized using a home-made reaction setup on gold-coated and non-coated Si (100) substrates at $950{ }^{\circ} \mathrm{C}$. XRD, SEM, EDX, and PL measurements were used for characterizations and it was found that a deposition duration of 10 minutes produced the most well-defined $\mathrm{ZnO}$ nanowires. SEM analysis revealed that the nanowires had diameters ranging from 30-100 $\mathrm{mm}$ and lengths ranging from 1-4 $\mu \mathrm{m}$. In addition, PL analysis showed strong UV emission at $380 \mathrm{~nm}$, making it suitable for UV lasing. Next, RF plasma was introduced for 30 minutes. Both remote and in situ RF plasma produced less satisfactory $\mathrm{ZnO}$ nanostructures with poorer crystalline structure, surface morphology, and optical properties due to etching effect of energetic ions produced from plasma. However, a reduction in plasma discharge duration to 10 minutes produced thicker and shorter $\mathrm{ZnO}$ nanostructures. Based on experimentation conducted, it is insufficient to conclude that RF plasma cannot aid in producing well-defined $\mathrm{ZnO}$ nanostructures. It can be deduced that the etching effect of energetic ions outweighed the increased oxygen radical production in RF plasma nanofabrication.
\end{abstract}

Keywords: ZnO nanowires; RF plasma; CVD.

This is an Open Access article published by World Scientific Publishing Company. It is distributed under the terms of the Creative Commons Attribution 3.0 (CC-BY) License. Further distribution of this work is permitted, provided the original work is properly cited. 


\section{Introduction}

Among all metal oxide nanoparticles, zinc oxide, $\mathrm{ZnO}$, stands out as one of the most versatile materials due to its diverse properties and functionalities. $\mathrm{ZnO}$ has a wide band gap of $3.37 \mathrm{eV}$ which makes it a very suitable semiconductor material. In addition, it has a high binding exciton energy of $60 \mathrm{meV}$ at room temperature (RT) and pressure. ${ }^{1}$ Due to these unique properties, there has been increasing studies done on $\mathrm{ZnO}$ nanostructures, leading to its applications in many different areas such as chemical and biological sensors, solar cells, light emitting diodes, drug delivery, lasers and composite materials. ${ }^{2}$

$\mathrm{ZnO}$ nanostructures have unique optical, electrical, mechanical and chemical properties due to their extremely small size which causes quantum confinement effects. $\mathrm{ZnO}$ has intrinsic n-type semi conductivity due to the presence of oxygen vacancies and/or zinc interstitials. ${ }^{3}$ Furthermore, $\mathrm{ZnO}$ nanostructures exhibit piezoelectric and pyroelectric properties due to a lack of center of symmetry in its wurtzite structure. ${ }^{4}$

$\mathrm{ZnO}$ nanostructures are commonly grown using thin film deposition methods, such as the vapor-liquid-solid (VLS) process, vapor transport method and chemical vapor deposition (CVD). ${ }^{5,6} \mathrm{CVD}$ is the process that leads to the formation of a non-volatile solid thin film on a substrate by the reaction of volatile chemical vapors that contain the required constituents. ${ }^{7}$ As deposition process is generally slower with direct adsorption of a gas phase onto a solid surface, VLS introduces a catalytic liquid (gold) which can increase the adsorption rates. ${ }^{8}$

Current literature has reported vertically well-aligned $\mathrm{ZnO}$ nanowires obtained by thermal evaporation with $350 \mathrm{sccm} \mathrm{Ar}_{-} \mathrm{O}_{2}$ gas flow rate with deposition duration of 90 minutes at $620{ }^{\circ} \mathrm{C}$. However, such long deposition duration makes it less favorable for mass industrial production. ${ }^{9}$ In other studies, well-aligned $\mathrm{Mn}$-doped $\mathrm{ZnO}$ nanostructures have been grown with radiofrequency (RF) plasma and they exhibited RT ferromagnetism properties. However the optical properties were undesirable with significant defects of oxygen vacancies ${ }^{10-14}$. RF power usage was also very high at $100 \mathrm{~W} .{ }^{15}$ Silicon nanowires have also been successfully grown at reduced deposition temperatures with the aid of RF plasma. ${ }^{16}$

Hence, this project aims to (i) optimize the operating conditions for growth of $\mathrm{ZnO}$ nanostructures using the CVD method, and (ii) investigate the effects of coupling radiofrequency $(\mathrm{RF})$ plasma to the $\mathrm{CVD}$ method on the quality of $\mathrm{ZnO}$ nanostructures.

\section{Research Method and Materials}

\subsection{Preparation of nanocrystalline $\mathrm{ZnO}$ powder}

$\mathrm{ZnO}$ powder was synthesized using the wet chemical method by reacting $3.95 \mathrm{~g}$ $(90 \mathrm{mMol})$ of zinc acetate dihydrate $\left[\mathrm{Zn}\left(\mathrm{CH}_{3} \mathrm{COO}\right)_{2} \cdot 2 \mathrm{H}_{2} \mathrm{O}\right]$ with $3.14 \mathrm{~g}(280 \mathrm{mMol})$ of potassium hydroxide $(\mathrm{KOH})$ in $50 \mathrm{ml}$ of methanol as reported in previous study. ${ }^{12}$ The contents were magnetically stirred continuously for 3 hours at $65^{\circ} \mathrm{C}$. Precipitates formed were then left to settle at room temperature $\left(25^{\circ} \mathrm{C}\right)$ for 24 hours and then washed 5 times 
with deionized water and removed by filtration. They were then dried at $45{ }^{\circ} \mathrm{C}$ to obtain solid powder.

\subsection{Optimization of operating conditions for $\mathrm{ZnO}$ nanostructures without $\mathrm{RF}$ plasma}

A home-made experimental setup was designed for RF plasma coupled CVD of ZnO nanostructures (Fig. 1). Heating zone of $25 \mathrm{~cm}$ in length was designed by coiling nickel chromium wire around the $70 \mathrm{~cm}$ long quartz tube with $22 \mathrm{~mm}$ outer diameter and $20 \mathrm{~mm}$ inner diameter. Glass fiber insulation cable was coiled over the wires for insulation.

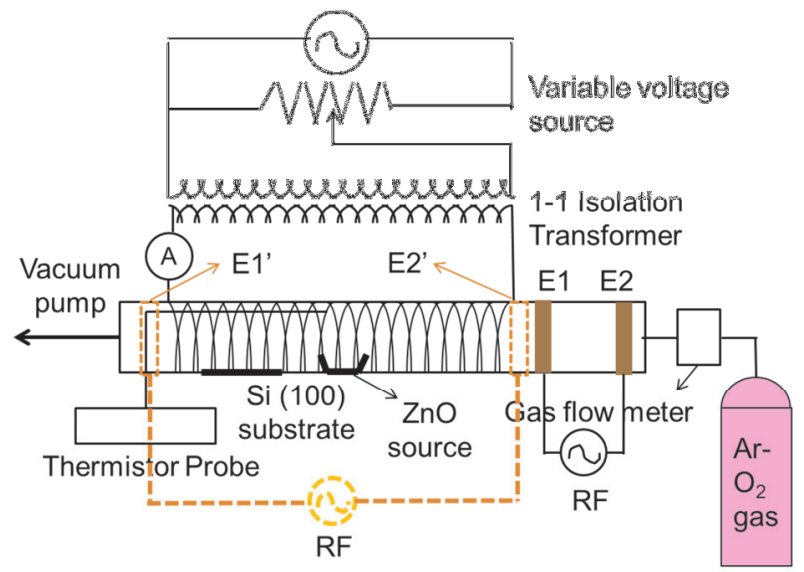

Fig. 1. Schematic diagram of experimental setup.

Temperature calibration was performed to determine which voltage and current values would give a temperature of $950{ }^{\circ} \mathrm{C}$ at the center of the heating zone in the quartz tube. Temperature recorded on the thermistor probe (placed outside the quartz reactor chamber) was also calibrated with respect to temperature inside the reactor tube as thermistor probe could not be placed in the reactor zone during the $\mathrm{ZnO}$ deposition.

$\mathrm{ZnO}$ source was prepared by mixing $0.5 \mathrm{~g}$ of $\mathrm{ZnO}$ powder with $0.5 \mathrm{~g}$ of graphite powder in 1:1 mass ratio and placed in an alumina boat in the center of the heating zone. $\mathrm{Si}$ (100) substrates of $3 \mathrm{~cm}$ by $0.5 \mathrm{~cm}$ dimensions were prepared and cleaned in an ultrasonic bath with acetone, followed by ethanol and deionized water at $45^{\circ} \mathrm{C}$, each with 15 minutes duration. Half of the cleaned substrates were then coated with a thin film of gold using the gold sputtering machine, at $20 \mathrm{~mA}$ for 30 seconds. Two Si (100) substrates, one with gold coating and one without gold coating were then placed in the experimental setup as shown, with center of the substrate at $11 \mathrm{~cm}$ away from the $\mathrm{ZnO}$ source.

After the experimental system was evacuated with a rotary pump, heater was turned on and voltage was gradually increased to increase the temperature of the heater. Ar- $\mathrm{O}_{2}$ $\left(90 \% \mathrm{Ar}, 10 \% \mathrm{O}_{2}\right)$ carrier gas started to flow when temperature of the thermistor probe 
recorded $600{ }^{\circ} \mathrm{C}$ with gas flow rate of $25 \mathrm{sccm}$ and background pressure of $2.0 \mathrm{mBar}$. Deposition timing was started once the temperature reached $950{ }^{\circ} \mathrm{C}$. Deposition duration for $\mathrm{ZnO}$ nanostructures was varied, at 2, 5, 10, 15 and 30 minutes, in a series of experiments to optimize the operating conditions for $\mathrm{ZnO}$ nanostructure growth.

\subsection{Effect of RF plasma on $\mathrm{ZnO}$ nanostructures}

$\mathrm{ZnO}$ nanostructure deposition was performed at $950{ }^{\circ} \mathrm{C}$ to investigate the effects of RF plasma on $\mathrm{ZnO}$ nanostructures, by using a commercial $13.56 \mathrm{MHz}$ RF generator (600 W, Cesar 136) with other conditions kept the same as above. RF plasma was turned on when temperature of the thermistor probe recorded $600{ }^{\circ} \mathrm{C}$, with plasma discharge duration of 30 minutes. Remote plasma at $950{ }^{\circ} \mathrm{C}$ was created with two RF electrodes (Fig. 1, E1, E2) separated by a distance of $4 \mathrm{~cm}$ with the first electrode $3 \mathrm{~cm}$ away from the heating zone. Following which, the distance between the electrodes was reduced to $2 \mathrm{~cm}$ with the first electrode $4 \mathrm{~cm}$ away from the heating zone as $\mathrm{ZnO}$ nanostructures could not be obtained initially. Gas flow rate and background pressure were then reduced to $20 \mathrm{sccm}$ and $1.0 \mathrm{mBar}$, respectively. In-situ plasma was then experimented with, at $950{ }^{\circ} \mathrm{C}$ and $900{ }^{\circ} \mathrm{C}$, by placing both electrodes (Fig. 1, E1', E2') $3 \mathrm{~cm}$ away from the heating zone. Lastly, plasma discharge duration was reduced from 30 to 10 minutes.

\subsection{Characterization of $\mathrm{ZnO}$ thin film samples}

The crystallinity of $\mathrm{ZnO}$ thin film samples were analyzed using SIEMENS D5005 Cu K $\alpha$ $(1.5406 \AA)$ X-Ray Diffractometer (XRD). Surface morphology and elemental composition of the thin films were characterized using the Jeol JSM 6700 Field Emission Scanning Electron Microscope (FESEM) and Energy Dispersive X-ray Spectroscopy (EDX). Lastly, the near band edge (NBE) and deep level emission (DLE) energy transitions were investigated using $\mathrm{He}-\mathrm{Cd}(325 \mathrm{~nm}, 10 \mathrm{~mW})$ to determine the photoluminescence (PL) spectra to investigate the structural defects in the $\mathrm{ZnO}$ samples at RT $\left(25^{\circ} \mathrm{C}\right)$.

\section{Results and Discussion}

\subsection{Optimization of operating conditions for $\mathrm{ZnO}$ nanostructures without $\mathrm{RF}$ plasma}

\subsubsection{Crystalline quality}

$\mathrm{XRD}$ spectra of $\mathrm{ZnO}$ thin film samples ${ }^{*}$ grown with various deposition durations without plasma are shown in Fig. 2a for gold-coated samples $(\mathrm{G})$ and Fig. $2 \mathrm{~b}$ for non-coated samples (NG).

\footnotetext{
${ }^{*}$ Refer to Appendix A for sample legend
} 

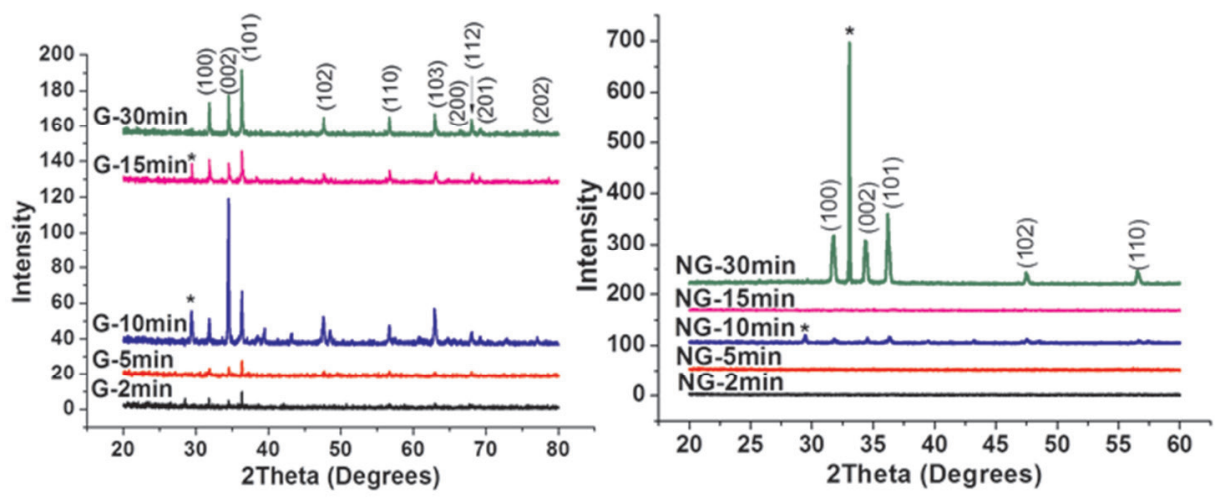

Fig. 2. XRD spectra of (a) gold-coated $\mathrm{ZnO}$ thin films (G) grown with various deposition durations (colour lines) without plasma (b) non-coated $\mathrm{ZnO}$ thin films (NG) grown with various deposition durations (colour lines) without plasma at $950^{\circ} \mathrm{C}$.

The crystallinity of gold-coated samples $(\mathrm{G})$ was found to be better than that of the non-coated samples (NG) as more material was deposited, allowing characterization to be performed with higher accuracy. In the gold-coated samples, impurity peaks were observed (asterisked) and this might be due to the deposition of zinc in its elemental form. Nonetheless, XRD diffraction patterns of gold-coated samples reflected most of the $\mathrm{ZnO}$ peaks, indicating successful growth of crystalline $\mathrm{ZnO}$ nanostructures. However in the non-coated samples, crystalline $\mathrm{ZnO}$ nanostructures were only shown in NG-10min and NG-30min samples, and there were also impurities observed in both the samples. The NG-10min sample was found to have a better crystallinity as its XRD diffraction pattern reflected more of the $\mathrm{ZnO}$ peaks as compared to NG-30min sample.

Based on calculated texture coefficients of peaks, diffraction peak intensity appeared to be the strongest at the (002) peak, indicating c-oriented growth of $\mathrm{ZnO}$ nanostructures. The average crystalline size was then calculated using Scherrer's equation with the full width half maximum (FWHM) value of (100), (002) and (101) peaks. There was no linear relationship between the average crystalline size and deposition duration (Fig. 3). The average crystalline size for some of the non-coated samples could not be calculated as they did not exhibit the (100), (002) and (101) peaks. G-5min appears to give the best crystalline size despite poor XRD diffraction patterns. However G-10min which appears to give better XRD diffraction patterns only produced $\mathrm{ZnO}$ with crystalline size of $21.4 \mathrm{~nm}$. Nonetheless, it can be observed that 10 minutes of deposition produces the best results, as the crystallinity of both the gold-coated and non-coated samples were comparatively better. 


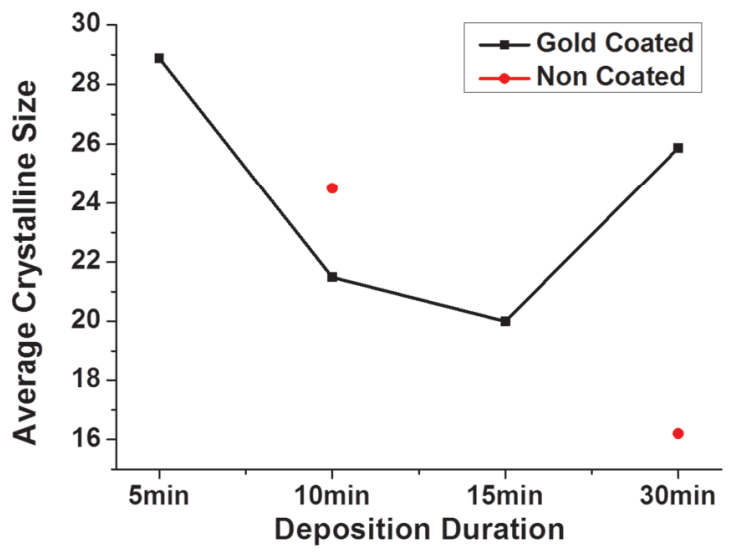

Fig. 3. Graph depicting variation between average crystalline size and deposition duration of gold-coated and non-coated $\mathrm{ZnO}$ thin film samples.

The lattice parameters, $a$ and $c$ of the $\mathrm{ZnO}$ thin film samples were then calculated using the (100) and (002) peaks (Fig. 4). Similarly, the lattice parameters for some of the non-coated samples could not be calculated as they did not exhibit the (100) and (002) peaks. Lattice parameter $a$ obtained for all samples except NG-30min appeared to be lower than that reported in literature $(a=3.2488 \AA)$, while lattice parameter $c$ obtained for all samples except G-5min were found to be similar to that reported in literature $(c=$ $5.2024 \AA$ A). It can be observed that the lattice parameters for both G-10min and NG-10min were relatively similar, unlike other deposition durations.

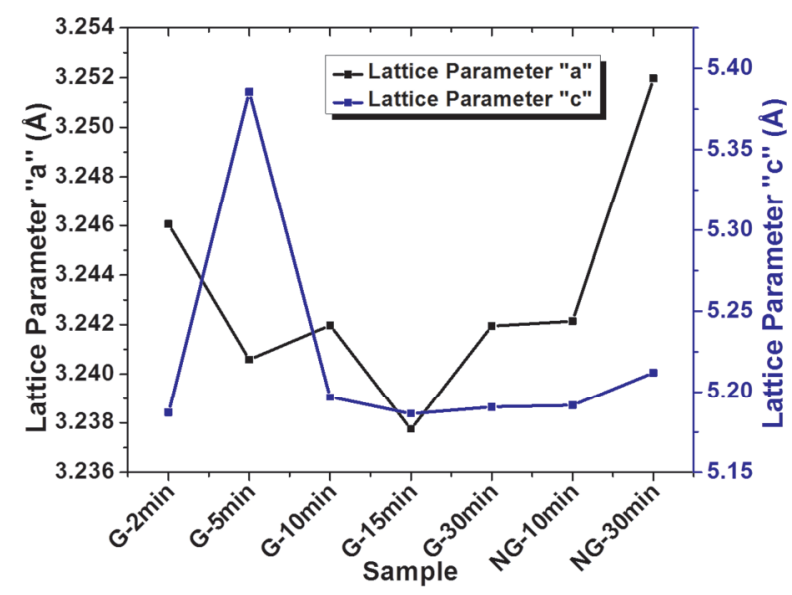

Fig. 4: Variation of lattice parameters "a" and "c" with respect to the different deposition durations for gold and non-coated $\mathrm{ZnO}$ thin film samples. 


\subsubsection{Surface morphology}

From Fig. 5, it can be observed that gold-coated substrates have material deposited on the entire substrate surface, with relatively high density of $\mathrm{ZnO}$ nanowires throughout the substrate as gold acts as a catalyst to enhance the deposition of $\mathrm{ZnO}$ nanowires.

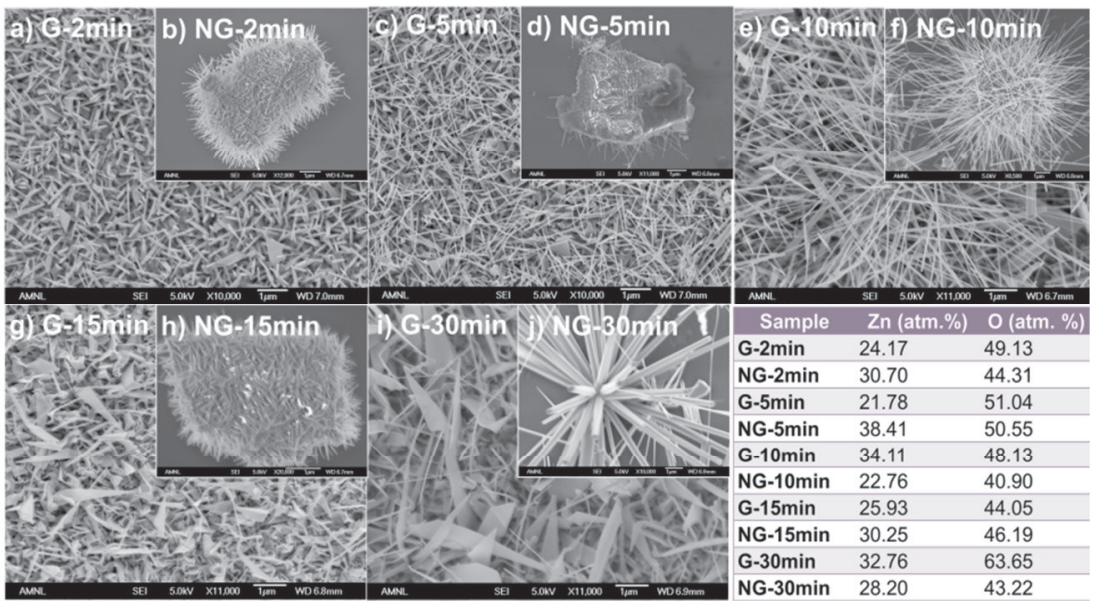

Fig. 5. FESEM micrographs of gold-coated and non-coated samples with various deposition durations along with the elemental analysis using EDX.

Deposition durations of 10 and 30 minutes were able to produce better $\mathrm{ZnO}$ nanowires and nanorods. Even though $\mathrm{ZnO}$ nanowires were obtained with the aid of gold catalyst in 2, 5 and 15 minutes deposition, well-defined nanowires were not formed without gold catalyst.

In G-30min, the length of the nanorods obtained ranged from $400 \mathrm{~nm}$ to a few microns, but most were found to be less than $1 \mu \mathrm{m}$. This was due to the formation of nanoplates that inhibited the vertical growth of nanorods. Nanoplates formed due to the long deposition duration increased the concentration of $\mathrm{Zn}$ and $\mathrm{O}$ vapor in the system and hence higher rates of nucleation and condensation of $\mathrm{ZnO}$.

A reduction in deposition time from 30 minutes to 10 minutes allowed for more nanowires to be obtained. In G-10min, nanowires were more well-defined and uniform. The average length of the nanowires ( 1 to $4 \mu \mathrm{m}$ ) increased, while the diameter of the nanowires in G-10min was relatively similar to that on G-30min.

In the non-coated samples, both NG-10min and NG-30min seemed to produce nanoflowers, where they were found to grow in clusters radiating spherically from a point. Nonetheless, the diameter of nanorods in NG-30min ranged from 200 to $500 \mathrm{~nm}$, while the diameter of nanowires in NG-10min ranged from 40 to $120 \mathrm{~nm}$. Nanowires were also found to have a hexagonal diameter in NG-10min which exhibited the structural properties of $\mathrm{ZnO}$, whereas nanorods had a circular diameter in NG-30min. 
Hence, this shows that a deposition duration of 10 minutes produces the best $\mathrm{ZnO}$ nanostructure surface features with smaller diameter and longer length of the nanowires, with and without gold catalyst.

\subsubsection{Optical properties}

The optical properties were studied using PL spectroscopy (Fig. 6). UV emission peaks $(\sim 381 \mathrm{~nm})$ were observed in all the samples due to the NBE emission of excitons. For the gold-coated samples, the intensity of UV emission was found to be relatively proportional to the deposition duration. For the non-coated samples, NG-30min gave the highest intensity of UV emission due to the large amount of material deposited, followed by NG-10min. DLE mainly due to the presence of oxygen vacancies was observed in all the samples. The defects were reduced and oxygen interstitials were not present with longer deposition duration.
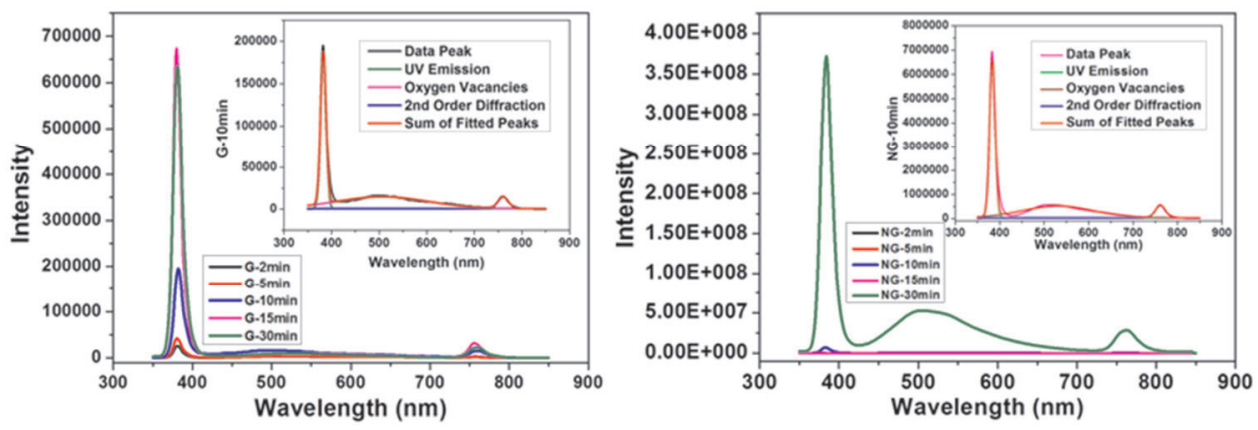

Fig. 6. RT PL spectra of (a) gold-coated $\mathrm{ZnO}$ thin film samples with deconvoluted peaks for G-10min (b) noncoated $\mathrm{ZnO}$ thin film samples with deconvoluted peaks for $\mathrm{NG}-10 \mathrm{~min}$.

The PL ratio of the samples was calculated by taking the percentage intensity of NBE over the percentage intensity of DLE to determine which samples have the best optical properties (Fig. 7). For the gold-coated samples, G-30min was found to have the best optical properties as it has the highest PL ratio. NG-2min was found to have the best optical properties as it has the highest PL ratio, followed by NG-10min. Hence it can be seen that even though 10 minute deposition duration produces the best surface features of $\mathrm{ZnO}$ nanowires, the optical quality of the thin film sample is reduced due to the presence of oxygen vacancies. 


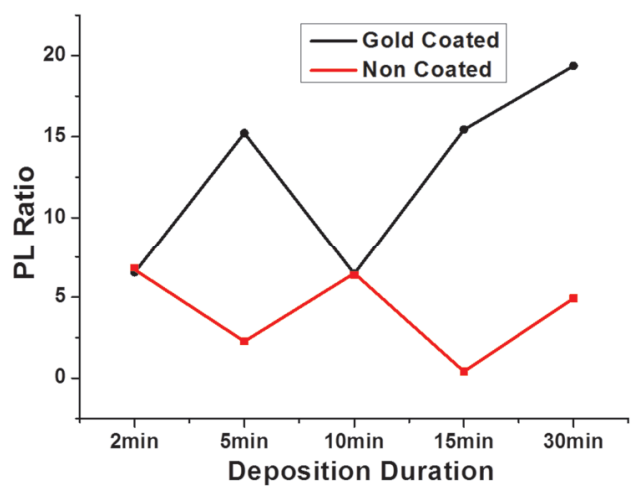

Fig. 7. PL ratio of gold-coated and non-coated $\mathrm{ZnO}$ thin film samples.

\subsection{Effect of RF plasma on $\mathrm{ZnO}$ nanostructures}

\subsubsection{Crystalline quality}

$\mathrm{XRD}$ spectra for the gold-coated and non-coated $\mathrm{ZnO}$ thin film samples ${ }^{\dagger}$ grown with $\mathrm{RF}$ plasma are shown in Fig. 8.

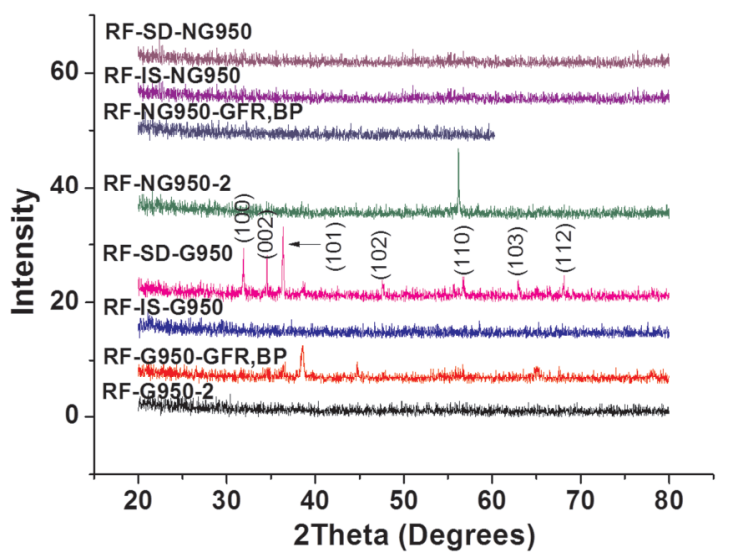

Fig. 8. XRD spectra of gold-coated and non-coated $\mathrm{ZnO}$ thin films grown with various deposition conditions (color lines) with RF plasma at $950^{\circ} \mathrm{C}$.

As can be seen from the XRD spectra, only RF-SD-G950 ZnO thin film sample managed to produce $\mathrm{ZnO}$ nanostructures with crystalline quality. Impurity peaks could be observed for other samples. Besides that, insufficient material deposited on the substrate could have also resulted in the lack of crystalline quality from the XRD spectra. The average crystalline size of RF-SD-G950 was $29.2 \mathrm{~nm}$, while the lattice parameters of $a$ was $3.238 \AA$ and $c$ was $5.189 \AA$ which are relatively similar to the standard lattice 
constant. However the average crystalline size is larger than the $\mathrm{ZnO}$ samples obtained from 10 minute deposition without RF plasma by about $7 \mathrm{~nm}$, and the lattice constants also differs more.

\subsubsection{Surface morphology}

From Fig. 9, it can be observed that both remote and in situ plasma deposition with RF plasma discharge of 30 minutes was unable to produce well-defined $\mathrm{ZnO}$ nanostructures. However plasma discharge of 10 minutes for remote plasma was able to produce $\mathrm{ZnO}$ nanostructures once again.

\begin{tabular}{|c|c|c|c|c|c|}
\hline G950-2 (b) RF-NG950-2 & $\begin{array}{l}\text { c) } \\
6950\end{array}$ & $\begin{array}{l}\text { d) RF-NG950- } \\
\text { GFR,BR }\end{array}$ & & & \\
\hline & & 0 & Sample & Zn (atm.\%) & $\mathrm{O}$ (atm.\%) \\
\hline & & & RF-G950-2 & 7.91 & 24.78 \\
\hline & & & RF-NG950-2 & 22.62 & 41.91 \\
\hline RF-IS- fी RF-IS-NG950 & ôRF-SD:- & h) RF-SD. & RF-G950-GFR,BP & 20.08 & 45.05 \\
\hline & & & RF-NG950-GFR,BP & 31.03 & 49.54 \\
\hline & & & RF-IS-G950 & 34.81 & 54.84 \\
\hline & & & RF-IS-NG950 & 42.78 & 55.44 \\
\hline & & & RF-SD-G950 & 16.23 & 23.88 \\
\hline & & & RF-SD-NG950 & 23.68 & 35.50 \\
\hline
\end{tabular}

Fig. 9. FESEM micrographs of gold-coated and non-coated samples with various deposition conditions in the presence of RF plasma, along with the elemental analysis using EDX.

With 30 minutes plasma discharge, small amounts of $\mathrm{ZnO}$ nanostructures were obtained with remote plasma, whereas in situ plasma was unable to produce nanostructures. This could be due to the fact that energetic ions from plasma etched away the nanostructures deposited on the substrate, whereas in remote plasma, the plasma was not formed across the substrate. Furthermore, by reducing the distance between the RF electrodes from $4 \mathrm{~cm}$ to $2 \mathrm{~cm}$, small amounts of nanostructures (Fig. 9a) were obtained. With a shorter plasma discharge distance, fewer energetic ions were produced. Hence less energetic ions would be present to etch away the $\mathrm{ZnO}$ nanostructures deposited on the substrates and less damage was done to the $\mathrm{ZnO}$ nanostructures, enabling nanostructures to be grown successfully.

$\mathrm{ZnO}$ nanostructures obtained with plasma discharge of 10 minutes had a thicker diameter (40 to $200 \mathrm{~nm}$ ) than those obtained without RF plasma, while the length of the nanorods and nanowires were shorter $(0.2$ to $4 \mu \mathrm{m})$. This shows that even though RF plasma was able to produce nanostructures, the surface features of the nanostructures were not as uniform and well-defined as compared to deposition without RF plasma. 


\subsubsection{Optical properties}

UV emission peaks ( $382 \mathrm{~nm}$ ) were observed in all the samples (Fig. 10), and majority of the samples showed DLE peaks of oxygen vacancies. DLE peaks were found to be almost equivalent or stronger than the NBE peaks, unlike in samples grown without RF plasma, where NBE peaks were much stronger. RF-G950-GFR,BP was the only exception, showing that with reduced gas flow rate $(20 \mathrm{sccm})$ and reduced background pressure (1.0 mBar), oxygen vacancies are reduced and $\mathrm{ZnO}$ with better optical properties are obtained.

As such, this shows that with the presence of RF plasma, the optical properties of the samples grown are reduced due to more oxygen vacancies. This could be due to the effect of energetic ions damaging the structure by etching away oxygen atoms which are more volatile as compared to zinc. Hence even though more oxygen radicals were produced, the damage done by energetic ions outweighed the increase oxygen radicals hence causing oxygen vacancies.

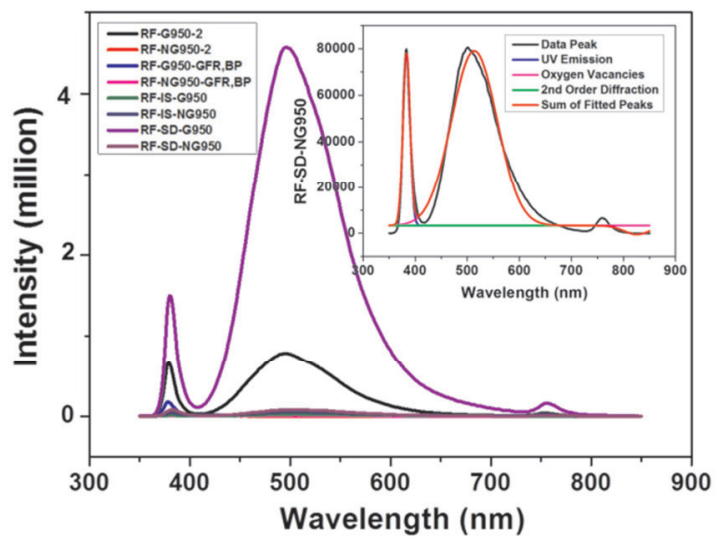

Figure 10. RT PL spectra of gold-coated and non-coated $\mathrm{ZnO}$ thin film samples with RF plasma deposited at $950{ }^{\circ} \mathrm{C}$ together with the deconvoluted peaks for RF-SD-NG950.

\section{Conclusion}

Well-defined and crystalline $\mathrm{ZnO}$ nanostructures were grown with deposition duration of 10 minutes without RF plasma. These $\mathrm{ZnO}$ nanostructures proved to have relatively good optical properties with high PL ratio of 19.4:1 for G-30min (NBE:DLE), indicating that there are minimal defect sites in the optical bandgap. However, $\mathrm{ZnO}$ nanostructures grown with RF plasma discharge of 10 minutes did not produce nanostructures that were as well-defined and crystalline. There were also defect sites in the optical bandgap, and poor electrical conductivity. Based on experimentation conducted, it is insufficient to conclude that RF plasma cannot aid in producing well-defined $\mathrm{ZnO}$ nanostructures. This is because even though the RF plasma discharge would have increased the oxygen radicals, which are essential in producing well-defined $\mathrm{ZnO}$ nanostructures, etching effect 
of argon ions had greater impact by removing the oxygen atoms from $\mathrm{ZnO}$, leading to oxygen deficient $\mathrm{ZnO}$. Hence, further studies would include varying the $\mathrm{Ar}: \mathrm{O}_{2}$ ratio in the precursor gas to reduce the detrimental etching effects of energetic ions on nanostructures, and also enhancing the positive effects of increased oxygen radicals to produce longer and well-defined $\mathrm{ZnO}$ nanostructures.

\section{Appendix A.}

\begin{tabular}{|l|l|}
\hline Sample & Description \\
\hline G-2min & Gold-coated $\mathrm{ZnO}$ thin film sample deposited for 2 minutes \\
\hline NG-2min & Non-coated $\mathrm{ZnO}$ thin film sample deposited for 2 minutes \\
\hline G-5min & Gold-coated $\mathrm{ZnO}$ thin film sample deposited for 5 minutes \\
\hline NG-5min & Non-coated $\mathrm{ZnO}$ thin film sample deposited for 5 minutes \\
\hline G-10min & Gold-coated $\mathrm{ZnO}$ thin film sample deposited for 10 minutes \\
\hline NG-10min & Non-coated $\mathrm{ZnO}$ thin film sample deposited for 10 minutes \\
\hline G-15min & Gold-coated $\mathrm{ZnO}$ thin film sample deposited for 15 minutes \\
\hline NG-15min & Non-coated $\mathrm{ZnO}$ thin film sample deposited for 15 minutes \\
\hline G-30min & Gold-coated ZnO thin film sample deposited for 30 minutes \\
\hline NG-30min & Non-coated $\mathrm{ZnO}$ thin film sample deposited for 30 minutes \\
\hline
\end{tabular}

\section{Appendix B.}

\begin{tabular}{|l|l|}
\hline Sample & Description \\
\hline RF-G950-2 & $\begin{array}{l}\text { Gold-coated } \mathrm{ZnO} \text { thin film sample deposited at } 950^{\circ} \mathrm{C} \text { with remote } \\
\text { RF plasma }\end{array}$ \\
\hline RF-NG950-2 & $\begin{array}{l}\text { Non-coated } \mathrm{ZnO} \text { thin film sample deposited at } 950^{\circ} \mathrm{C} \text { with remote } \\
\text { RF plasma }\end{array}$ \\
\hline $\begin{array}{l}\text { RF-G950- } \\
\text { GFR,BP }\end{array}$ & $\begin{array}{l}\text { Gold-coated } \mathrm{ZnO} \text { thin film sample deposited at } 950^{\circ} \mathrm{C} \text { with remote } \\
\text { RF plasma with reduced gas flow rate and background pressure }\end{array}$ \\
\hline $\begin{array}{l}\text { RF-NG950- } \\
\text { GFR,BP }\end{array}$ & $\begin{array}{l}\text { Non-coated } \mathrm{ZnO} \text { thin film sample deposited at } 950^{\circ} \mathrm{C} \text { with remote } \\
\text { RF plasma with reduced gas flow rate and background pressure }\end{array}$ \\
\hline RF-IS-G950 & $\begin{array}{l}\text { Gold-coated } \mathrm{ZnO} \text { thin film sample deposited at } 950^{\circ} \mathrm{C} \text { with in situ } \\
\text { RF plasma }\end{array}$ \\
\hline RF-IS-NG950 & $\begin{array}{l}\text { Non-coated } \mathrm{ZnO} \text { thin film sample deposited at } 950^{\circ} \mathrm{C} \text { with in situ } \\
\text { RF plasma }\end{array}$ \\
\hline RF-SD-G950 & $\begin{array}{l}\text { Gold-coated } \mathrm{ZnO} \text { thin film sample deposited at } 950^{\circ} \mathrm{C} \text { with remote } \\
\text { RF plasma with shorter duration (10min) for plasma exposure }\end{array}$ \\
\hline RF-SD-NG950 & $\begin{array}{l}\text { Non-coated } \mathrm{ZnO} \text { thin film sample deposited at } 950^{\circ} \mathrm{C} \text { with remote } \\
\text { RF plasma with shorter duration (10min) for plasma exposure }\end{array}$ \\
\hline
\end{tabular}




\section{Acknowledgments}

I would like to express my utmost gratitude to Dr Usman Ilyas for the help rendered in training me in the usage of various equipments, and for his invaluable advice on the project. I would also like to thank Dr Chen Rui ${ }^{\ddagger}$ for his assistance in performing the photoluminescence analysis of all the $\mathrm{ZnO}$ thin film samples, Dr Rajdeep for his supervision throughout the course of the project. Lastly, I would like to thank Ms Loh Yuhui from River Valley High School, Singapore for her support and valuable comments for the project.

\section{References}

1. Z. L. Wang Journal of Physics: Condensed Matter 16, 829 (2004).

2. Ming Wei, J. L. MacManus-Driscoll Journal of Physics: Conference Series 26, 300 (2006).

3. J. Goldbereger, D. J. Sirbuly, M. Law et al. The Journal of Physical Chemistry 109, 9 (2005).

4. Z. Y. Fan, J.G. Lu Journal of Nanoscience and Nanotechnology 10, 1561 (2005).

5. Y.M. Wu, X.J. Zhang, H. H. Pan, et al., Scientific Reports 3, 3248 (2013).

6. R.S. Wagner, W.C. Ellis Applied Physics Letters 4, 89 (1964).

7. T.H. Cui, Oxidation and CVD, from http://www.me.umn.edu/courses/me8254/attfiles/ Lecture\%2008\%200xidation\%20and\%20CVD.pdf.

8. G.C. Yi, Semiconductor Nanostructures for Optoelectronic Devices, (Germany, 2012).

9. Tran Van Khai, Sin Young Bang, Dong Keun Oh et al., Journal of Ceramic Processing Research 12, 106 (2011).

10. U. Ilyas, R. S. Rawat, T. L. Tan, P. Lee, R. Chen, H. D. Sun, L. Fengji and S. Zhang, J. Appl. Phys. 111, 33503 (2012).

11. U. Ilyas, R. S. Rawat, Y. Wang, T. L. Tan, P. Lee, R. Chen, H. D. Sun, F. Li and S. Zhang, Appl. Surf. Sci. 258, 6373 (2012).

12. U. Ilyas, R. S. Rawat, G. Roshan, T. L. Tan, P. Lee, S. V. Springham, S. Zhang, L. Fengji, R. Chen and H. D. Sun, Appl. Surf. Sci. 258, 890 (2011).

13. U. Ilyas, R. S. Rawat, T. L. Tan, P. Lee, R. Chen, H. D. Sun, L. Fengji and S. Zhang, J. Appl. Phys. 110, 093522 (2011).

14. U. Ilyas, T. L. Tan, P. Lee, R. V. Ramanujan, Fengji Li, Sam Zhang, R. Chen, H. D. Sun, R. S. Rawat, Journal of Magnetism and Magnetic Materials 344, 171 (2013).

15. Z. F. Wu, X.M. Wu, L. J. Zhuge et al., Materials Letters 64, 472 (2010).

16. S. Hofmann, C. Ducati, J. Neill et al., Journal of Applied Physics 94, 6005 (2003).

\footnotetext{
${ }^{\ddagger}$ School of Physical and Mathematical Sciences, Nanyang Technological University.
} 